Journal of Mathematical Physics, Analysis, Geometry

2015, vol. 11, No. 3, pp. 279-296

\title{
On the Jost Solutions for a Class of Schrödinger Equations with Piecewise Constant Coefficients
}

\author{
A.A. Nabiev \\ Department of Mathematics, Faculty of Science \\ Cumhuriyet University, Sivas, 58140 Turkey \\ E-mail: aadiloglu@cumhuriyet.edu.tr \\ Kh.R. Mamedov \\ Department of Mathematics, Faculty of Science and Letters \\ Mersin University, Mersin, 33343 Turkey \\ E-mail: hanlar@mersin.edu.tr
}

Received October 23, 2013, revised August 03, 2014

In this paper, the new integral representations for the Jost solutions of the one-dimensional Schrödinger equation with the piecewise-constant leading coefficient are obtained. The connections, obtained between the kernel functions of the integral representations and the potential function of the Schrödinger equation, enable to solve the inverse scattering problem on the entire real line.

Key words: Schrödinger equation, Jost solution, integral representation.

Mathematics Subject Classification 2010: 34L40.

\section{Introduction}

Consider the one dimensional Schrödinger equation with the piecewise-constant leading coefficient

$$
-y^{\prime \prime}+q(x) y=\lambda^{2} \rho(x) y, \quad-\infty<x<\infty,
$$

where $\lambda$ is a spectral parameter, $q(x)$ is a complex valued function satisfying the condition

$$
\int_{-\infty}^{\infty}(1+x)|q(x)| d x<\infty
$$

and

$$
\rho(x)=\left\{\begin{array}{cc}
\alpha^{2}, & x<a, \\
1, & x \geq a,
\end{array}\right.
$$

(C) A.A. Nabiev and Kh.R. Mamedov, 2015 
$0<\alpha \neq 1, a \in(-\infty,+\infty)$.

In the case $\alpha=1$, that is, when $\rho(x) \equiv 1$, there are well-known 'triangular' integral representations for the Jost solutions of equation (1.1) satisfying the conditions at infinity (see [1], [2], [5], [8], etc). The Jost solutions play an important role in the studying of the inverse scattering problem on the whole real line (see $[3],[7],[6],[4])$.

It turns out that in the case $\rho(x) \neq 1$, the function $\rho(x)$ strongly influences on the structure of the integral representations of the solutions and the main integral equations of Marchenko-Faddeev's type of the inverse scattering problem for equation (1.1). Note that the inverse scattering problem for equation (1.1) on the half-line $[0, \infty)$, with discontinuity at any point $a(0<a<\infty)$, was solved in [9], [10].

In the present paper, under conditions (1.2), (1.3) we construct new (nontriangular) integral representations of the Jost solutions of equation (1.1) on the whole real line. The properties of the Jost solutions are studied and some significant connections are obtained between the potential $q(x)$ of equation (1.1) and the kernel of the integral representation. These connections reveal that the inverse scattering problem for equation (1.1) can be solved by using the new integral representations of the Jost solutions.

\section{Integral Representation of the Jost Solutions}

Without loss of generality, one can consider that a point of discontinuity of the function $\rho(x)$ coincides with the origin, i.e., $a=0$.

We denote by $f_{ \pm}(x, \lambda)$ the solution of equation (1.1) with the condition

$$
\lim _{x \rightarrow \pm \infty} f_{ \pm}(x, \lambda) e^{\mp i \lambda \mu(x)}=1,
$$

where $\mu(x)=x \sqrt{\rho(x)}$. The solutions $f_{+}(x, \lambda)$ and $f_{-}(x, \lambda)$ will be called the right and the left Jost solutions of equation (1.1), respectively. It is easy to verify that the solutions $f_{ \pm}(x, \lambda)$ obey the integral equations

$$
\begin{aligned}
f_{ \pm}(x, \lambda) & =e_{ \pm}(x, \lambda)+\int_{x}^{ \pm \infty}\left[\frac{1}{2}\left(\frac{1}{\sqrt{\rho(t)}}-\frac{1}{\sqrt{\rho(x)}}\right) \frac{\sin \lambda(\mu(t)+\mu(x))}{\lambda}\right. \\
+ & \left.\frac{1}{2}\left(\frac{1}{\sqrt{\rho(t)}}+\frac{1}{\sqrt{\rho(x)}}\right) \frac{\sin \lambda(\mu(t)-\mu(x))}{\lambda}\right] q(t) f_{ \pm}(t, \lambda) d t
\end{aligned}
$$

where

$$
e_{+}(x, \lambda)=\frac{1}{2}\left(1+\frac{1}{\sqrt{\rho(x)}}\right) e^{i \lambda \mu(x)}+\frac{1}{2}\left(1-\frac{1}{\sqrt{\rho(x)}}\right) e^{-i \lambda \mu(x)}
$$


and

$$
e_{-}(x, \lambda)=\frac{1}{2}\left(1-\frac{\alpha}{\sqrt{\rho(x)}}\right) e^{i \lambda \mu(x)}+\frac{1}{2}\left(1+\frac{\alpha}{\sqrt{\rho(x)}}\right) e^{-i \lambda \mu(x)} .
$$

Consider the solution $f_{+}(x, \lambda)$. When $x>0$, it is known that (see [7]) for all $\operatorname{Im} \lambda \geq 0$ the solution $f_{+}(x, \lambda)$ has the representation

$$
f_{+}(x, \lambda)=e^{i \lambda x}+\int_{x}^{+\infty} A^{+}(x, t) e^{i \lambda t} d t
$$

where the function $A^{+}(x, t)$ and its first-order partial derivatives satisfy the inequalities

$$
\begin{gathered}
\left|A^{+}(x, t)\right| \leq \frac{1}{2} \sigma^{+}\left(\frac{x+t}{2}\right) e^{\sigma_{1}^{+}(x)-\sigma_{1}^{+}\left(\frac{x+t}{2}\right)} \\
\left|\frac{\partial A^{+}\left(x_{1}, x_{2}\right)}{\partial x_{i}}+\frac{1}{4} q\left(\frac{x_{1}+x_{2}}{2}\right)\right| \leq \frac{1}{2} \sigma_{1}^{+}\left(x_{1}\right) \sigma^{+}\left(\frac{x_{1}+x_{2}}{2}\right) e^{\sigma_{1}(x)}
\end{gathered}
$$

in which $\sigma_{1}^{+}(x)=\int_{x}^{+\infty} \sigma^{+}(t) d t$ and $\sigma^{+}(x)=\int_{x}^{+\infty}|q(t)| d t$. Moreover,

$$
\begin{gathered}
A^{+}(x, x)=\frac{1}{2} \int_{x}^{+\infty} q(t) d t \\
A^{+}(x, t)=\frac{1}{2} \int_{\frac{x+t}{2}}^{+\infty} q(s) d s+\int_{\frac{x+t}{2}}^{+\infty} d u \int_{0}^{\frac{x-t}{2}} q(u-v) A^{+}(u-v, u+v) d v, t>x,
\end{gathered}
$$

and $A^{+}(x, t) \equiv 0$ for $t<x$.

Now we study the interesting case $x<0$. In this case, equation (2.1) takes the form

$$
\begin{gathered}
f_{+}(x, \lambda)=\alpha_{+} e^{i \alpha \lambda x}+\alpha_{-} e^{-i \alpha \lambda x}+\int_{x}^{0} \frac{\sin \alpha \lambda(t-x)}{\alpha \lambda} q(t) f_{+}(t, \lambda) d t \\
+\int_{0}^{\infty}\left[\alpha^{-\frac{\sin \lambda(t+\alpha x)}{\lambda}}+\alpha^{+} \frac{\sin \lambda(t-\alpha x)}{\lambda}\right] q(t) f_{+}(t, \lambda) d t,
\end{gathered}
$$

where $\alpha^{ \pm}=\frac{1}{2}\left(1 \pm \frac{1}{\alpha}\right)$. We require that the solution of the integral equation (2.6) be of the form

$$
f_{+}(x, \lambda)=\alpha_{+} e^{i \alpha \lambda x}+\alpha_{-} e^{-i \alpha \lambda x}+\int_{\alpha x}^{+\infty} B^{+}(x, t) e^{i \lambda t} d t, \operatorname{Im} \lambda \geq 0, x<0,
$$


where $B^{+}(x, t)$ will be defined below. Substituting $f_{+}(x, \lambda)$ in equation (2.6) with the representation

$$
f_{+}(x, \lambda)=e^{i \lambda x}+\int_{x}^{+\infty} A^{+}(x, t) e^{i \lambda t} d t
$$

for $x>0$ and the representation (2.7) for $x<0$, after some transformations we find that

$$
\begin{gathered}
B^{+}(x, t)=\frac{\alpha^{+}}{2 \alpha} \int_{\frac{t+\alpha x}{2 \alpha}}^{0} q(s) d s+\frac{\alpha^{-}}{2 \alpha} \int_{\frac{\alpha x-t}{2 \alpha}}^{0} q(s) d s+\frac{\alpha^{+}}{2} \int_{0}^{+\infty} q(s) d s \\
-\frac{\alpha^{-}}{2} \int_{0}^{\frac{t-\alpha x}{2}} q(s) d s+\alpha^{+} \int_{0}^{\frac{t-\alpha x}{2}} d v \int_{v}^{+\infty} q(u-v) A^{+}(u-v, u+v) d u \\
-\alpha^{-} \int_{0}^{\frac{t-\alpha x}{2}} d v \int_{v}^{\frac{t-\alpha x}{2}} q(u-v) A^{+}(u-v, u+v) d u \\
+\frac{1}{\alpha^{2}} \int_{0}^{\frac{t-\alpha x}{2}} d v \int_{\frac{t+\alpha x}{2}}^{v}\left(\frac{u-v}{\alpha}\right) B^{+}\left(\frac{u-v}{\alpha}, u+v\right) d u, \quad \alpha x \leq t<-\alpha x \\
B^{+}(x, t)=\frac{\alpha^{+}}{2} \int_{\frac{t+\alpha x}{2}}^{+\infty} q(s) d s+\frac{\alpha^{-}}{2} \int_{\frac{t-\alpha x}{2}}^{+\infty} q(s) d s \\
+\alpha^{+} \int_{\frac{t+\alpha x}{2}}^{\frac{t-\alpha x}{2}} d v \int_{v}^{+\infty} q(u-v) A^{+}(u-v, u+v) d u \\
+\alpha^{+} \int_{0}^{\frac{t+\alpha x}{2}} d v \int_{\frac{t+\alpha x}{2}}^{+\infty} q(u-v) A^{+}(u-v, u+v) d u \\
+\alpha^{-} \int_{0}^{\frac{t+\alpha x}{2}} d v \int_{\frac{t-\alpha x}{2}}^{+\infty} q(u-v) A^{+}(u-v, u+v) d u \\
-\alpha^{-} \int_{\frac{t+\alpha x}{2}}^{\frac{t-\alpha x}{2}} d v \int_{v}^{\frac{t-\alpha x}{2}} q(u-v) A^{+}(u-v, u+v) d u \\
d v \int_{\frac{t+\alpha x}{2}}^{v}\left(\frac{u-v}{\alpha}\right) B^{+}\left(\frac{u-v}{\alpha}, u+v\right) d v, t>-\alpha x .
\end{gathered}
$$

Here we suppose $A^{+}(x, t) \equiv 0$ for $t<x$ and $B^{+}(x, t) \equiv 0$ for $t<\alpha x$. have

In the similar way, considering the solution $f_{-}(x, \lambda)$, for the case $x<0$, we

$$
f_{-}(x, \lambda)=e^{-i \alpha \lambda x}+\int_{-\infty}^{\alpha x} A^{-}(x, t) e^{-i \lambda t} d t, \operatorname{Im} \lambda \geq 0, x<0,
$$


where

$$
A^{-}(x, t)=\frac{1}{2 \alpha} \int_{-\infty}^{\frac{\alpha x+t}{2 \alpha}} q(s) d s+\int_{-\infty}^{\frac{\alpha x+t}{2 \alpha}} d u \int_{0}^{\frac{\alpha x-t}{2 \alpha}} q(u+v) A^{-}(u+v, \alpha(u-v)) d v
$$

and $A^{-}(x, t) \equiv 0$ for $t>\alpha x$. It is easy to verify that

$$
\begin{gathered}
\left|A^{-}(x, t)\right| \leq \frac{1}{2 \alpha} \sigma^{-}\left(\frac{\alpha x+t}{2 \alpha}\right) e^{\sigma_{1}^{-}(x)-\sigma_{1}^{-}\left(\frac{\alpha x+t}{2 \alpha}\right)} \\
\left|\frac{\partial A^{-}(x, t)}{\partial x}-\frac{1}{4 \alpha} q\left(\frac{\alpha x+t}{2 \alpha}\right)\right| \leq \frac{1}{2 \alpha} \sigma^{-}\left(\frac{\alpha x+t}{2 \alpha}\right) \sigma^{-}(x) e^{\sigma_{1}^{-}(x)-\sigma_{1}^{-}\left(\frac{\alpha x+t}{2 \alpha}\right)}, \\
\left|\frac{\partial A^{-}(x, t)}{\partial t}-\frac{1}{4 \alpha^{2}} q\left(\frac{\alpha x+t}{2 \alpha}\right)\right| \leq \frac{1}{2 \alpha^{2}} \sigma^{-}\left(\frac{\alpha x+t}{2 \alpha}\right) \sigma^{-}(x) e^{\sigma_{1}^{-}(x)-\sigma_{1}^{-}\left(\frac{\alpha x+t}{2 \alpha}\right)}
\end{gathered}
$$

and

$$
A^{-}(x, \alpha x)=\frac{1}{2 \alpha} \int_{-\infty}^{x} q(s) d s,
$$

where $\sigma^{-}(x)=\int_{-\infty}^{x}|q(s)| d s, \sigma_{1}^{-}(x)=\int_{-\infty}^{x} \sigma^{-}(s) d s$. As in the case of the right Jost solution, for $x>0$, we have

$f_{-}(x, \lambda)=\frac{1}{2}(1-\alpha) e^{i \lambda x}+\frac{1}{2}(1+\alpha) e^{-i \lambda x}+\int_{-\infty}^{x} B^{-}(x, t) e^{-i \lambda t} d t, \operatorname{Im} \lambda \geq 0, x>0$,

where

$$
\begin{gathered}
B^{-}(x, t)=\frac{\alpha^{-}}{2} \int_{\frac{t-x}{2 \alpha}}^{0} q(s) d s+\frac{\alpha^{+}}{2} \int_{-\infty}^{0} q(s) d s+\frac{1}{4}(1+\alpha) \int_{0}^{\frac{x+t}{2}} q(s) d s \\
+\frac{1}{4}(1-\alpha) \int_{0}^{\frac{x-t}{2}} q(s) d s+\alpha \alpha^{-} \int_{0}^{\frac{x-t}{2 \alpha}} d v \int_{\frac{t-x}{2 \alpha}}^{-v} q(u+v) A^{-}(u+v, \alpha(u-v)) d u \\
+\alpha \alpha^{+} \int_{0}^{\frac{x-t}{2 \alpha}} d v \int_{-\infty}^{-v} q(u+v) A^{-}(u+v, \alpha(u-v)) d u \\
+\int_{0}^{\frac{x-t}{2}} d v \int_{-v}^{\frac{x+t}{2}} q(u+v) B^{-}(u+v, u-v) d u,-x<t \leq x, x>0
\end{gathered}
$$




$$
\begin{aligned}
& B^{-}(x, t)= \frac{\alpha^{+}}{2} \int_{-\infty}^{\frac{t+x}{2 \alpha}} q(s) d s-\frac{\alpha^{-}}{2} \int_{-\infty}^{\frac{t-x}{2 \alpha}} q(s) d s \\
&+\alpha^{-} \alpha \int_{-\frac{x+t}{2 \alpha}}^{\frac{x-t}{2 \alpha}} d v \int_{-\frac{x-t}{2 \alpha}}^{-v} q(u+v) A^{-}(u+v, \alpha(u-v)) d u \\
&+\alpha^{-} \alpha \int_{0}^{-\frac{x+t}{2 \alpha}} d v \int_{-\infty}^{\frac{t-x}{2 \alpha}} q(u+v) A^{-}(u+v, \alpha(u-v)) d u \\
&+\alpha \alpha^{+} \int_{0}^{-\frac{x+t}{2 \alpha}} d v \int_{-\infty}^{\frac{x+t}{2 \alpha}} q(u+v) A^{-}(u+v, \alpha(u-v)) d u \\
&+\alpha \alpha^{+} \int_{-\frac{x+t}{2 \alpha}}^{\frac{x-t}{2 \alpha}} d v \int_{-\infty}^{-v} q(u+v) A^{-}(u+v, \alpha(u-v)) d u \\
&+\int_{-\frac{x+t}{2}}^{\frac{x-t}{2}} d v \int_{-v}^{\frac{x+t}{2}} q(u+v) B^{-}(u+v, u-v) d u, t<-x<0,
\end{aligned}
$$

and $B^{-}(x, t) \equiv 0$ for $t>x$.

Now we can solve the system of integral equations (2.8) and (2.9). Setting $B^{+}(x, t)=F^{+}(r, p), \alpha x+t=2 r, t-\alpha x=2 p(x<0)$, from (2.8) and (2.9), we have

$$
\begin{gathered}
F^{+}(r, p)=\frac{\alpha^{+}}{2 \alpha} \int_{\frac{r}{\alpha}}^{0} q(u) d u+\frac{\alpha^{-}}{2 \alpha} \int_{-\frac{p}{\alpha}}^{0} q(v) d v+\frac{\alpha^{+}}{2} \int_{0}^{+\infty} q(s) d s-\frac{\alpha^{-}}{2} \int_{0}^{p} q(u) d u \\
+\alpha^{+} \int_{0}^{p} d v \int_{v}^{+\infty} q(u-v) H^{+}(u, v) d u-\alpha^{-} \int_{0}^{p} d v \int_{v}^{p} q(u-v) H^{+}(u, v) d u \\
+\frac{1}{\alpha^{2}} \int_{0}^{p} d v \int_{r}^{v} q\left(\frac{u-v}{\alpha}\right) F^{+}(u, v) d u, r<0 \leq p, \quad(2.13) \\
F^{+}(r, p)=\frac{\alpha^{+}}{2} \int_{r}^{+\infty} q(s) d s+\frac{\alpha^{-}}{2} \int_{p}^{+\infty} q(s) d s \\
+\alpha^{+} \int_{r}^{p} d v \int_{v}^{+\infty} q(u-v) H^{+}(u, v) d u+\alpha^{+} \int_{0}^{r} d v \int_{r}^{+\infty} q(u-v) H^{+}(u, v) d u \\
+\alpha^{-} \int_{0}^{r} d v \int_{p}^{+\infty} q(u-v) H^{+}(u, v) d u-\alpha^{-} \int_{r}^{p} d v \int_{v}^{p} q(u-v) H^{+}(u, v) d u \\
+\frac{1}{\alpha^{2}} \int_{r}^{p} d v \int_{r}^{v} q\left(\frac{u-v}{\alpha}\right) F^{+}(u, v) d u, 0<r \leq p,
\end{gathered}
$$

here $H^{+}(u, v)=A^{+}(u-v, u+v),(u \geq v \geq 0)$. 
Let

$$
\begin{gathered}
\varphi^{+}(r, p)=\frac{\alpha^{+}}{2} \int_{r}^{+\infty} q(s) d s+\frac{\alpha^{-}}{2} \int_{p}^{+\infty} q(s) d s \\
+\alpha^{+} \int_{r}^{p} d v \int_{v}^{+\infty} q(u-v) H^{+}(u, v) d u \\
+\alpha^{+} \int_{0}^{r} d v \int_{r}^{+\infty} q(u-v) H^{+}(u, v) d u \\
+\alpha^{-} \int_{0}^{r} d v \int_{p}^{+\infty} q(u-v) H^{+}(u, v) d u \\
-\alpha^{-} \int_{r}^{p} d v \int_{v}^{p} q(u-v) H^{+}(u, v) d u, \quad 0<r \leq p,
\end{gathered}
$$

and

$$
\begin{aligned}
\varphi^{+}(r, p)= & \frac{\alpha^{+}}{2 \alpha} \int_{\frac{r}{\alpha}}^{0} q(u) d u+\frac{\alpha^{-}}{2 \alpha} \int_{-\frac{p}{\alpha}}^{0} q(v) d v+\frac{\alpha^{+}}{2} \int_{0}^{+\infty} q(s) d s \\
& -\frac{\alpha^{-}}{2} \int_{0}^{p} q(u) d u+\alpha^{+} \int_{0}^{p} d v \int_{v}^{+\infty} q(u-v) H^{+}(u, v) d u \\
- & \alpha^{-} \int_{0}^{p} d v \int_{v}^{p} q(u-v) H^{+}(u, v) d u, \quad r<0 \leq p .
\end{aligned}
$$

Then equations (2.13) and (2.14) can be written as

$$
F^{+}(r, p)=\varphi^{+}(r, p)+\frac{1}{\alpha^{2}} \int_{\max (0, r)}^{p} d v \int_{r}^{v} q\left(\frac{u-v}{\alpha}\right) F^{+}(u, v) d u, r \leq p, p \geq 0 .
$$

From inequality (2.4) we have

$$
\left|H^{+}(u, v)\right| \leq \frac{1}{2} \sigma^{+}(u) e^{\sigma_{1}^{+}(u-v)-\sigma_{1}^{+}(u)} .
$$

Using this, it is easy to obtain

$$
\left|\varphi^{+}(r, p)\right| \leq \frac{C_{0}}{2} \sigma^{+}(r) e^{\sigma_{1}^{+}(r-p)-\sigma_{1}^{+}(r)}, 0<r \leq p,
$$

and

$$
\left|\varphi^{+}(r, p)\right| \leq C_{0}^{2} \sigma^{+}\left(\min \left(\frac{r}{\alpha},-\frac{p}{\alpha}\right)\right) e^{\sigma_{1}^{+}(0)-\sigma_{1}^{+}(p)}, r<0 \leq p
$$

where $C_{0}=\max \left(1, \frac{1}{\alpha}\right)$. Now we define the successive approximations for $F^{+}(r, p)$ as

$$
F_{0}^{+}(r, p)=\varphi^{+}(r, p)
$$




$$
F_{m}^{+}(r, p)=\frac{1}{\alpha^{2}} \int_{\max (0, r)}^{p} d v \int_{r}^{v} q\left(\frac{u-v}{\alpha}\right) F_{m-1}^{+}(u, v) d u, r \leq p, p \geq 0 .
$$

Using estimation (2.16) and (2.17), for $m=0,1,2, \ldots$, we immediately have

$$
\left|F_{m}^{+}(r, p)\right| \leq \frac{C_{0}}{2} \sigma^{+}(r) e^{\sigma_{1}^{+}(r-p)-\sigma_{1}^{+}(r)} \frac{\left[\tau^{+}\left(\frac{r-p}{\alpha}\right)\right]^{m}}{m !}, 0<r \leq p
$$

and

$$
\left|F_{m}^{+}(r, p)\right| \leq C_{0}^{2} \sigma^{+}\left(\min \left(\frac{r}{\alpha},-\frac{p}{\alpha}\right)\right) e^{\sigma_{1}^{+}(0)-\sigma_{1}^{+}(p)} \frac{\left[\tau^{+}\left(\frac{r-p}{\alpha}\right)\right]^{m}}{m !}, r<0 \leq p,
$$

where $\tau^{+}(x)=\int_{x}^{0} \sigma^{+}(s) d s$. These estimations imply that the integral equations (2.13) and (2.14) have the unique solution $F^{+}(r, p)$, and

$$
\begin{gathered}
\left|F^{+}(r, p)\right| \leq \frac{C_{0}}{2} \sigma^{+}(r) e^{\sigma_{1}^{+}(r-p)-\sigma_{1}^{+}(r)} e^{\tau^{+}\left(\frac{r-p}{\alpha}\right)}, 0<r \leq p, \\
\left|F^{+}(r, p)\right| \leq C_{0}^{2} \sigma^{+}\left(\min \left(\frac{r}{\alpha},-\frac{p}{\alpha}\right)\right) e^{\sigma_{1}^{+}(0)-\sigma_{1}^{+}(p)} e^{\tau^{+}\left(\frac{r-p}{\alpha}\right)}, r<0 \leq p,
\end{gathered}
$$

are satisfied.

Returning to the variables $x, t$, we can find

$$
\begin{gathered}
\left|B^{+}(x, t)\right| \leq \frac{C_{0}}{2} \sigma^{+}\left(\frac{\alpha x+t}{2}\right) e^{\sigma_{1}^{+}(\alpha x)-\sigma_{1}^{+}\left(\frac{t+\alpha x}{2}\right)+\tau^{+}(x)}, t>-\alpha x, \\
\left|B^{+}(x, t)\right| \leq C_{0}^{2} \sigma^{+}\left(\frac{\alpha x+t}{2 \alpha}\right) e^{\sigma_{1}^{+}(0)-\sigma_{1}^{+}\left(\frac{t-\alpha x}{2}\right)+\tau^{+}(x)}, \alpha x<t<0, \\
\left|B^{+}(x, t)\right| \leq C_{0}^{2} \sigma^{+}\left(\frac{\alpha x-t}{2 \alpha}\right) e^{\sigma_{1}^{+}(0)-\sigma_{1}^{+}\left(\frac{t-\alpha x}{2}\right)+\tau^{+}(x)}, 0<t<-\alpha x .
\end{gathered}
$$

Analogously, from inequality (2.10) we have that if we define

$$
H^{-}(r, p)=A^{-}(r+p, \alpha(r-p)), r<-p<0,
$$

then

$$
\left|H^{-}(r, p)\right| \leq \frac{1}{2 \alpha} \sigma^{-}(r) e^{\sigma_{1}^{-}(r+p)-\sigma_{1}^{-}(r)} .
$$

Let $B^{-}(x, t)=F^{-}(r, p), x+t=2 r, x-t=2 p(x<0)$ in equations $(2.11)$ and (2.12). Then we have

$$
\begin{aligned}
F^{-}(r, p)= & \frac{\alpha^{-}}{2} \int_{-\frac{p}{\alpha}}^{0} q(u) d u+\frac{\alpha^{+}}{2} \int_{-\infty}^{0} q(u) d u+\frac{1}{2} \alpha \alpha^{+} \int_{0}^{r} q(u) d u \\
& -\frac{1}{2} \alpha \alpha^{-} \int_{0}^{p} q(u) d u+\alpha \alpha^{+} \int_{0}^{\frac{p}{\alpha}} d v \int_{-\infty}^{-v} q(u+v) H^{-}(u, v) d u \\
& +\alpha \alpha^{-} \int_{0}^{\frac{p}{\alpha}} d v \int_{-\frac{p}{\alpha}}^{-v} q(u+v) H^{-}(u, v) d u
\end{aligned}
$$




$$
\begin{gathered}
+\int_{0}^{p} d v \int_{-v}^{r} q(u+v) F^{-}(u, v) d u, \quad r>0, \quad p \geq 0, \\
F^{-}(r, p)=\frac{\alpha^{+}}{2} \int_{-\infty}^{\frac{r}{\alpha}} q(u) d u-\frac{\alpha^{-}}{2} \int_{-\infty}^{-\frac{p}{\alpha}} q(u) d u \\
+\alpha^{-} \alpha \int_{-\frac{r}{\alpha}}^{\frac{p}{\alpha}} d v \int_{-\frac{p}{\alpha}}^{-v} q(u+v) H^{-}(u, v) d u-\alpha^{-} \alpha \int_{0}^{-\frac{r}{\alpha}} d v \int_{-\infty}^{-\frac{p}{\alpha}} q(u+v) H^{-}(u, v) d u \\
+\alpha \alpha^{+} \int_{0}^{-\frac{r}{\alpha}} d v \int_{-\infty}^{\frac{r}{\alpha}} q(u+v) H^{-}(u, v) d u+\alpha \alpha^{+} \int_{-\frac{r}{\alpha}}^{\frac{p}{\alpha}} d v \int_{-\infty}^{-v} q(u+v) H^{-}(u, v) d u \\
+\int_{-r}^{p} d v \int_{-v}^{r} q(u+v) F^{-}(u, v) d u, \quad-p<r<0 \leq p .
\end{gathered}
$$

We also define

$$
\begin{aligned}
\varphi^{-}(r, p)= & \frac{\alpha^{-}}{2} \int_{-\frac{p}{\alpha}}^{0} q(u) d u+\frac{\alpha^{+}}{2} \int_{-\infty}^{0} q(u) d u-\frac{1}{2} \alpha \alpha^{+} \int_{0}^{r} q(u) d u \\
& -\frac{1}{2} \alpha \alpha^{-} \int_{0}^{p} q(u) d u+\alpha \alpha^{+} \int_{0}^{\frac{p}{\alpha}} d v \int_{-\infty}^{-v} q(u+v) H^{-}(u, v) d u \\
& +\alpha \alpha^{+} \int_{0}^{\frac{p}{\alpha}} d v \int_{-\frac{p}{\alpha}}^{-v} q(u+v) H^{-}(u, v) d u, \quad r>0, \quad p \geq 0,
\end{aligned}
$$

and

$$
\begin{aligned}
\varphi^{-}(r, p)= & \frac{\alpha^{+}}{2} \int_{-\infty}^{\frac{r}{\alpha}} q(u) d u-\frac{\alpha^{-}}{2} \int_{-\infty}^{-\frac{p}{\alpha}} q(u) d u \\
& +\alpha \alpha^{-} \int_{-\frac{r}{\alpha}}^{\frac{p}{\alpha}} d v \int_{-\frac{p}{\alpha}}^{-v} q(u+v) H^{-}(u, v) d u \\
& -\alpha^{-} \alpha \int_{0}^{-\frac{r}{\alpha}} d v \int_{-\infty}^{-\frac{p}{\alpha}} q(u+v) H^{-}(u, v) d u \\
& +\alpha \alpha^{+} \int_{0}^{-\frac{r}{\alpha}} d v \int_{-\infty}^{\frac{r}{\alpha}} q(u+v) H^{-}(u, v) d u \\
& +\alpha \alpha^{+} \int_{-\frac{r}{\alpha}}^{\frac{p}{\alpha}} d v \int_{-\infty}^{-v} q(u+v) H^{-}(u, v) d u, \quad-p<r<0 \leq p .
\end{aligned}
$$

Obviously,

$$
F^{-}(r, p)=\varphi^{-}(r, p)+\int_{\min (-r, 0)}^{p} d v \int_{-v}^{r} q(u+v) F^{-}(u, v) d u, p<r, p \geq 0
$$


Using estimation (2.23), we obtain

$$
\left|F^{-}(r, p)\right| \leq \frac{C_{0}}{2} \sigma^{-}\left(\frac{r}{\alpha}\right) e^{\sigma_{1}^{-}\left(\frac{r+p}{\alpha}\right)-\sigma_{1}^{-}\left(\frac{r}{\alpha}\right)}+\tau^{-}\left(\frac{r+p}{\alpha}\right),-p<r<0 \leq p .
$$

If $r>0$, then

$$
\begin{aligned}
\left|\varphi^{-}(r, p)\right| \leq & \frac{\alpha C_{o}^{2}}{2} \int_{-\infty}^{\min (r, p)}|q(s)| d s \\
& +C_{0} \alpha \int_{-\frac{p}{\alpha}}^{0} d v \int_{0}^{-u}|q(u+v)|\left|H^{-}(u, v)\right| d v \\
& +C_{0} \alpha \int_{-\infty}^{-\frac{p}{\alpha}} d v \int_{0}^{\frac{p}{\alpha}}|q(u+v)|\left|H^{-}(u, v)\right| d v \\
\leq & \frac{\alpha C_{o}^{2}}{2} \sigma^{-}(\max (r, p)) \\
& +\frac{C_{0}}{2} \int_{-\frac{p}{\alpha}}^{0} d v \int_{0}^{-u}|q(u+v)| \sigma^{-}(u) e^{\sigma^{-}(u+v)-\sigma^{-}(u)} d v \\
& +\frac{C_{0}}{2} \int_{-\infty}^{-\frac{p}{\alpha}} d u \int_{0}^{\frac{p}{\alpha}}|q(u+v)| \sigma^{-}(u) e^{\sigma^{-}(u+v)-\sigma^{-}(u)} d v \\
\leq & \frac{\alpha C_{o}^{2}}{2} \sigma^{-}(\max (r, p)) \\
& +\frac{C_{0}}{2} \sigma^{-}(0) \int_{-\infty}^{0} e^{\sigma^{-}\left(u+\frac{p}{\alpha}\right)-\sigma^{-}(u)} d u \int_{u}^{\frac{u+p}{\alpha}}|q(s)| d s \\
\leq & \frac{\alpha C_{o}^{2}}{2} \sigma^{-}(\max (r, p)) e^{\sigma^{-}\left(\frac{p}{\alpha}\right)-\sigma^{-}(0)},
\end{aligned}
$$

i.e.,

$$
\left|\varphi^{-}(r, p)\right| \leq \frac{\alpha C_{o}^{2}}{2} \sigma^{-}(\max (r, p)) e^{\sigma^{-}\left(\frac{p}{\alpha}\right)-\sigma^{-}(0)}, \quad r>0, \quad p \geq 0 .
$$

Now using (2.27) and (2.28), from (2.26), we can obtain

$$
\left|F^{-}(r, p)\right| \leq \frac{C_{0}}{2} \sigma^{-}\left(\frac{r}{\alpha}\right) e^{\sigma_{1}^{-}\left(\frac{r+p}{\alpha}\right)-\sigma_{1}^{-}\left(\frac{r}{\alpha}\right)+\tau^{-}(r+p)},-p<r<0 \leq p,
$$

and

$$
\left|F^{-}(r, p)\right| \leq \frac{\alpha C_{0}}{2} \sigma^{-}(\max (r, p)) e^{\sigma_{1}^{-}\left(\frac{p}{\alpha}\right)-\sigma_{1}^{-}(0)+\tau^{-}(r+p)}, r>0, p \geq 0,
$$

where $\tau^{-}(x)=\int_{0}^{x} \sigma^{-}(s) d s$. 
Returning to the variables $x$ and $t$, from (2.29) and (2.30) we find

$$
\left|B^{-}(x, t)\right| \leq \alpha C_{o}^{2} \sigma^{-}\left(\frac{x+t}{2 \alpha}\right) e^{\sigma_{1}^{-}\left(\frac{x}{\alpha}\right)-\sigma_{1}^{-}\left(\frac{x+t}{2 \alpha}\right)+\tau^{-}(x)}, t<-x,
$$

and

$$
\left|B^{-}(x, t)\right| \leq \alpha C_{o}^{2} \sigma^{-}\left(\max \left(\frac{x+t}{2}, \frac{x-t}{2}\right)\right) e^{\sigma_{1}^{-}\left(\frac{x-t}{2 \alpha}\right)-\sigma_{1}^{-}(0)+\tau^{-}(x)},-x<t \leq x .
$$

Now, from (2.20)-(2.22) and (2.31), (2.32), we can easily obtain that

$$
\pm \int_{\alpha x}^{ \pm \infty}\left|B^{ \pm}(x, t)\right| d t \leq C\left\{e^{\sigma^{ \pm}(\alpha x)}-1\right\}
$$

for some $C>0$.

Hence, by setting $K^{ \pm}(x, t)=\left\{\begin{array}{ll}A^{ \pm}(x, t), & \pm x \geq 0 \\ B^{ \pm}(x, t), & \pm x<0\end{array}\right.$ and combining all our results, we can formulate the following theorem:

Theorem 1. If condition (1.3) is satisfied, then for every value of the parameter $\lambda$ from the upper half-plane $\operatorname{Im} \lambda \geq 0$ the Jost solutions $f_{ \pm}(x, \lambda)$ of discontinuous Schrödinger equation (1.1) have the representations

$$
f_{ \pm}(x, \lambda)=e_{ \pm}(x, \lambda) \pm \int_{\mu(x)}^{ \pm \infty} K^{ \pm}(x, t) e^{i \lambda t} d t
$$

where $e_{ \pm}(x, \lambda)$ are defined by (2.2), (2.3), $\mu(x)=x \sqrt{\rho(x)}$ and the kernels $K^{ \pm}(x, t)$ satisfy the inequalities

$$
\pm \int_{\mu(x)}^{ \pm \infty}\left|K^{ \pm}(x, t)\right| d t \leq C\left\{e^{\sigma^{ \pm}(\mu(x))}-1\right\}
$$

for some $C>0$. Moreover, the following relations are satisfied:

$$
\begin{gathered}
K^{+}(x, \mu(x))=\frac{1}{4}\left(1+\frac{1}{\sqrt{\rho(x)}}\right) \int_{x}^{+\infty} \frac{q(s)}{\sqrt{\rho(s)}} d s, \\
K^{+}(x,-\mu(x)+0)-K^{+}(x,-\mu(x)-0)=\frac{1}{4}\left(1-\frac{1}{\sqrt{\rho(x)}}\right) \int_{x}^{+\infty} \frac{\text { sgns }}{\sqrt{\rho(s)}} q(s) d s, \\
K^{-}(x, \mu(x))=\frac{1}{4}\left(1+\frac{\alpha}{\sqrt{\rho(x)}}\right) \int_{-\infty}^{x} \frac{q(s)}{\sqrt{\rho(s)}} d s, \\
K^{-}(x,-\mu(x)+0)-K^{-}(x,-\mu(x)-0)=\frac{1}{4}\left(1-\frac{\alpha}{\sqrt{\rho(x)}}\right) \int_{-\infty}^{x} \frac{\operatorname{sgns}}{\sqrt{\rho(s)}} q(s) d s .
\end{gathered}
$$


For further studying the properties of the kernel $K^{ \pm}(x, t)$ let us differentiate equations $(2.13),(2.14)$ and $(2.24),(2.25)$ with respect to both variables. We have

$$
\begin{aligned}
& \frac{\partial F^{+}(r, p)}{\partial r}=-\frac{\alpha^{+}}{2 \alpha} q\left(\frac{r}{\alpha}\right)-\frac{1}{\alpha^{2}} \int_{0}^{p} q\left(\frac{r-v}{\alpha}\right) F^{+}(r, v) d v, r<0 \leq p, \\
& \frac{\partial F^{+}(r, p)}{\partial r}=-\frac{\alpha^{+}}{2} q(r)-\alpha^{+} \int_{0}^{r} q(r-v) H^{+}(r, v) d v \\
& +\alpha^{-} \int_{r}^{+\infty} q(u-r) H^{+}(u, r) d u-\frac{1}{\alpha^{2}} \int_{r}^{p} q\left(\frac{r-v}{\alpha}\right) F^{+}(r, v) d v, 0<r \leq p \text {, } \\
& \frac{\partial F^{+}(r, p)}{\partial p}=\frac{\alpha^{-}}{2 \alpha^{2}} q\left(-\frac{p}{\alpha}\right)-\frac{\alpha^{-}}{2} q(p)+\alpha^{+} \int_{p}^{+\infty} q(u-p) H^{+}(u, p) d u \\
& \alpha^{-} \int_{0}^{p} q(p-v) H^{+}(p, v) d v+\frac{1}{\alpha^{2}} \int_{r}^{p} q\left(\frac{u-p}{\alpha}\right) F^{+}(u, p) d u, r<0 \leq p, \\
& \frac{\partial F^{+}(r, p)}{\partial p}=-\frac{\alpha^{-}}{2} q(p)+\alpha^{+} \int_{p}^{+\infty} q(u-p) H^{+}(u, p) d u \\
& -\alpha^{-} \int_{0}^{r} q(p-v) H^{+}(p, v) d v-\int_{r}^{p} q(p-v) H^{+}(p, v) d v \\
& +\frac{1}{\alpha^{2}} \int_{r}^{p} q\left(\frac{u-p}{\alpha}\right) F^{+}(u, p) d u, 0<r \leq p .
\end{aligned}
$$

Then, by using estimations $(2.15),(2.18),(2.19)$, it is easy to obtain from (2.38)(2.40) that

$$
\begin{gathered}
\left|\frac{\partial F^{+}(r, p)}{\partial r}+\frac{\alpha^{+}}{2 \alpha} q\left(\frac{r}{\alpha}\right)\right| \\
\leq M^{+} \sigma^{+}\left(\min \left(\frac{r}{\alpha},-\frac{p}{\alpha}\right)\right) e^{\sigma_{1}^{+}(r-p)-\sigma_{1}^{+}(r)+\tau^{+}\left(\frac{r-p}{\alpha}\right)}, r<0 \leq p, \\
\left|\frac{\partial F^{+}(r, p)}{\partial r}+\frac{\alpha^{+}}{2} q(r)\right| \leq M^{+} \sigma^{+}(r) e^{\sigma_{1}^{+}(r-p)-\sigma_{1}^{+}(r)+\tau^{+}\left(\frac{r-p}{\alpha}\right)}, 0<r \leq p, \\
\left|\frac{\partial F^{+}(r, p)}{\partial p}-\frac{\alpha^{-}}{2 \alpha^{2}} q\left(-\frac{p}{\alpha}\right)+\frac{\alpha^{-}}{2} q(p)\right| \\
\leq M^{+} \sigma^{+}\left(\min \left(\frac{r}{\alpha},-\frac{p}{\alpha}\right)\right) e^{\sigma_{1}^{+}(r-p)-\sigma_{1}^{+}(r)+\tau^{+}\left(\frac{r-p}{\alpha}\right)}, r<0 \leq p, \\
\left|\frac{\partial F^{+}(r, p)}{\partial p}-\frac{\alpha^{-}}{2 \alpha^{2}} q\left(-\frac{p}{\alpha}\right)+\frac{\alpha^{-}}{2} q(p)\right| \\
\leq M^{+} \sigma^{+}(r) e^{\sigma_{1}^{+}(r-p)-\sigma_{1}^{+}(r)+\tau^{+}\left(\frac{r-p}{\alpha}\right)}, 0<r \leq p,
\end{gathered}
$$


where $M^{+}$is some positive constant. From $(2.24),(2.25)$, in the similar way we obtain

$$
\begin{gathered}
\frac{\partial F^{-}(r, p)}{\partial r}=\frac{1}{2} \alpha \alpha^{+} q(r)+\int_{0}^{p} q(r+v) F^{-}(r, v) d v, r>0, p \geq 0 \\
\frac{\partial F^{-}(r, p)}{\partial r}=\frac{\alpha^{+}}{2 \alpha} q\left(\frac{r}{\alpha}\right)+\alpha^{-} \int_{-\infty}^{\frac{r}{\alpha}} q\left(u-\frac{r}{\alpha}\right) H^{-}\left(u,-\frac{r}{\alpha}\right) d u \\
+\alpha^{+} \int_{0}^{-\frac{r}{\alpha}} q\left(\frac{r}{\alpha}+v\right) H^{-}\left(\frac{r}{\alpha}, v\right) d v+\int_{-r}^{p} q(r+v) F^{-}(r, v) d u,-p<r<0 \leq p \\
\frac{\partial F^{-}(r, p)}{\partial p}=\frac{\alpha^{-}}{2 \alpha} q\left(-\frac{p}{\alpha}\right)-\frac{1}{2} \alpha \alpha^{-} q(p)+\alpha^{+} \int_{-\infty}^{-\frac{p}{\alpha}} q\left(u+\frac{p}{\alpha}\right) H^{-}\left(u, \frac{p}{\alpha}\right) d u \\
+\alpha^{-} \int_{0}^{\frac{p}{\alpha}} q\left(-\frac{p}{\alpha}+v\right) H^{-}\left(-\frac{p}{\alpha}, v\right) d v+\int_{-p}^{r} q(u+p) F^{-}(u, p) d u, r>0, p \geq 0 \\
\frac{\partial F^{-}(r, p)}{\partial p}=\frac{\alpha^{-}}{2 \alpha} q\left(-\frac{p}{\alpha}\right)+\alpha^{+} \int_{-\infty}^{-\frac{p}{\alpha}} q\left(u+\frac{p}{\alpha}\right) H^{-}\left(u, \frac{p}{\alpha}\right) d u \\
+\alpha^{-} \int_{0}^{\frac{p}{\alpha}} q\left(-\frac{p}{\alpha}+v\right) H^{-}\left(-\frac{p}{\alpha}, v\right) d v+\int_{-p}^{r} q(u+p) F^{-}(u, p) d u,-p<r<0 \leq p .
\end{gathered}
$$

Now estimations (2.23), (2.29), (2.30) imply that

$$
\begin{gathered}
\quad\left|\frac{\partial F^{-}(r, p)}{\partial r}-\frac{\alpha^{+} \alpha}{2} q(r)\right| \\
\leq M^{-} \sigma^{-}(\max (r, p)) e^{\sigma_{1}^{-}\left(\frac{r+p}{\alpha}\right)-\sigma_{1}^{-}\left(\frac{r}{\alpha}\right)+\tau^{-}(r+p)}, r>0, p \geq 0, \\
\left|\frac{\partial F^{-}(r, p)}{\partial r}-\frac{\alpha^{+}}{2 \alpha} q\left(\frac{r}{\alpha}\right)\right| \\
\leq M^{-} \sigma^{-}\left(\frac{r}{\alpha}\right) e^{\sigma_{1}^{-}\left(\frac{r+p}{\alpha}\right)-\sigma_{1}^{-}\left(\frac{r}{\alpha}\right)+\tau^{-}(r+p)},-p<r<0 \leq p, \\
\left|\frac{\partial F^{-}(r, p)}{\partial p}+\frac{\alpha^{-}}{2 \alpha} q\left(-\frac{p}{\alpha}\right)+\frac{\alpha^{-} \alpha}{2} q(p)\right| \\
\leq M^{-} \sigma^{-}(\max (r, p)) e^{\sigma_{1}^{-}\left(\frac{r+p}{\alpha}\right)-\sigma_{1}^{-}\left(\frac{r}{\alpha}\right)+\tau^{-}(r+p)}, r>0, p \geq 0, \\
\left|\frac{\partial F^{-}(r, p)}{\partial p}-\frac{\alpha^{-}}{2 \alpha} q\left(-\frac{p}{\alpha}\right)\right| \\
\leq M^{-} \sigma^{-}\left(\frac{r}{\alpha}\right) e^{\sigma_{1}^{-}\left(\frac{r+p}{\alpha}\right)-\sigma_{1}^{-}\left(\frac{r}{\alpha}\right)+\tau^{-}(r+p)},-p<r<0 \leq p .
\end{gathered}
$$


Furthermore, by differentiation, from (2.38), (2.39) and (2.45), ( 2.46) we have that $F^{+}(r, p)$ and $F^{-}(r, p)$ satisfy the partial differential equations

$$
\frac{\partial^{2} F^{+}(r, p)}{\partial p \partial r}=-\frac{1}{\alpha^{2}} q\left(\frac{r-p}{\alpha}\right) F^{+}(r, p)
$$

and

$$
\frac{\partial^{2} F^{-}(r, p)}{\partial p \partial r}=q(r+p) F^{-}(r, p)
$$

respectively. Since

$$
\begin{aligned}
& \frac{\partial B^{+}(x, t)}{\partial x}=\frac{\alpha}{2}\left(\frac{\partial F^{+}(r, p)}{\partial r}-\frac{\partial F^{+}(r, p)}{\partial p}\right), \\
& \frac{\partial B^{+}(x, t)}{\partial t}=\frac{1}{2}\left(\frac{\partial F^{+}(r, p)}{\partial r}+\frac{\partial F^{+}(r, p)}{\partial p}\right),
\end{aligned}
$$

from (2.41)-(2.44) we have

$$
\begin{gathered}
\left|\frac{\partial B^{+}(x, t)}{\partial x}+\frac{\alpha^{+}}{4 \alpha} q\left(\frac{\alpha x+t}{2 \alpha}\right)+\frac{\alpha^{-}}{4 \alpha} q\left(\frac{\alpha x-t}{2 \alpha}\right)-\frac{\alpha \alpha^{-}}{4} q\left(\frac{t-\alpha x}{2}\right)\right|, \\
\left|\frac{\partial B^{+}(x, t)}{\partial t}+\frac{\alpha^{+}}{4 \alpha^{2}} q\left(\frac{\alpha x+t}{2 \alpha}\right)+\frac{\alpha^{-}}{4 \alpha^{2}} q\left(\frac{\alpha x-t}{2 \alpha}\right)-\frac{\alpha^{-}}{4} q\left(\frac{t-\alpha x}{2}\right)\right| \\
\leq \alpha M^{+} \sigma^{+}\left(\min \left(\frac{\alpha x+t}{2 \alpha}, \frac{\alpha x-t}{2 \alpha}\right)\right) e^{\sigma_{1}^{+}(\alpha x)-\sigma_{1}^{+}\left(\frac{t+\alpha x}{2}\right)+\tau^{+}(x)}, \alpha x \leq t<-\alpha x, \\
\left|\frac{\partial B^{+}(x, t)}{\partial x}+\frac{\alpha^{+} \alpha}{4} q\left(\frac{\alpha x+t}{2}\right)-\frac{\alpha^{-} \alpha}{4} q\left(\frac{t-\alpha x}{2}\right)\right| \\
\leq \frac{\partial B^{+}(x, t)}{\partial t}+\frac{\alpha^{+}}{4} q\left(\frac{\alpha x+t}{2}\right)-\frac{\alpha^{-}}{4} q\left(\frac{t-\alpha x}{2}\right) \mid \\
\leq \alpha M^{+} \sigma^{+}\left(\frac{\alpha x+t}{2}\right) e^{\sigma_{1}^{+}(\alpha x)-\sigma_{1}^{+}\left(\frac{t+\alpha x}{2}\right)+\tau^{+}(x)}, t>-\alpha x .
\end{gathered}
$$

Analogously, because of the relations

$$
\begin{aligned}
& \frac{\partial B^{-}(x, t)}{\partial x}=\frac{1}{2}\left(\frac{\partial F^{-}(r, p)}{\partial r}+\frac{\partial F^{-}(r, p)}{\partial p}\right), \\
& \frac{\partial B^{-}(x, t)}{\partial t}=\frac{1}{2}\left(\frac{\partial F^{-}(r, p)}{\partial r}-\frac{\partial F^{-}(r, p)}{\partial p}\right),
\end{aligned}
$$

we obtain from (2.47)-(2.50) that

$$
\left|\frac{\partial B^{-}(x, t)}{\partial x}-\frac{\alpha \alpha^{+}}{4} q\left(\frac{x+t}{2}\right)-\frac{\alpha \alpha^{-}}{4} q\left(\frac{x-t}{2}\right)+\frac{\alpha^{-}}{4 \alpha} q\left(\frac{t-x}{2 \alpha}\right)\right|,
$$




$$
\begin{gathered}
\left|\frac{\partial B^{-}(x, t)}{\partial t}-\frac{\alpha \alpha^{+}}{4} q\left(\frac{x+t}{2}\right)+\frac{\alpha \alpha^{-}}{4} q\left(\frac{x-t}{2}\right)-\frac{\alpha^{-}}{4 \alpha} q\left(\frac{t-x}{2 \alpha}\right)\right| \\
\leq M^{-} \sigma^{-}\left(\max \left(\frac{x+t}{2}, \frac{x-t}{2}\right)\right) e^{\sigma_{1}^{-}\left(\frac{x}{\alpha}\right)-\sigma_{1}^{-}\left(\frac{x+t}{2 \alpha}\right)+\tau^{-}(x)}, \quad-x<t \leq x, \\
\left|\frac{\partial B^{-}(x, t)}{\partial x}-\frac{\alpha^{+}}{4 \alpha} q\left(\frac{x+t}{2 \alpha}\right)-\frac{\alpha^{-}}{4 \alpha} q\left(\frac{t-x}{2 \alpha}\right)\right|, \\
\quad\left|\frac{\partial B^{-}(x, t)}{\partial t}-\frac{\alpha^{+}}{4 \alpha} q\left(\frac{x+t}{2 \alpha}\right)+\frac{\alpha^{-}}{4 \alpha} q\left(\frac{t-x}{2 \alpha}\right)\right| \\
\leq M^{-} \sigma^{-}\left(\frac{x+t}{2 \alpha}\right) e^{\sigma_{1}^{-}\left(\frac{x}{\alpha}\right)-\sigma_{1}^{-}\left(\frac{x+t}{2 \alpha}\right)+\tau^{-}(x)}, t<-x .
\end{gathered}
$$

Note that from (2.14) we have

$$
F^{+}(p, p)=\frac{1}{2} \int_{p}^{+\infty} q(s) d s+\frac{1}{2} \int_{0}^{p} d v \int_{p}^{+\infty} q(u-v) H^{+}(u, v) d u,
$$

which yields

$$
F^{+}(p, p)=H^{+}(p, p)
$$

according to (2.5). Returning to the variables $x$ and $t$, we immediately have

$$
B^{+}(0, t)=A^{+}(0, t), t>0 .
$$

Similarly, from (2.25) and the integral equation for the kernel $A^{-}(x, t)$ (see the equation for $\left.A^{-}(x, t)\right)$, we obtain

$$
B^{-}(0, t)=A^{-}(0, t), t<0 .
$$

From $(2.39)$ and $\left(2.40^{\prime}\right)$, we also have

$$
\begin{aligned}
\frac{\partial F^{+}(p, p)}{\partial r} & =-\frac{\alpha^{+}}{2} q(p)-\alpha^{+} \int_{0}^{p} q(p-v) H^{+}(p, v) d v \\
& +\alpha^{-} \int_{p}^{+\infty} q(u-p) H^{+}(u, p) d u, \\
\frac{\partial F^{+}(p, p)}{\partial p}= & -\frac{\alpha^{-}}{2} q(p)+\alpha^{+} \int_{p}^{+\infty} q(u-p) H^{+}(u, p) d u \\
& -\alpha^{-} \int_{0}^{p} q(p-v) H^{+}(p, v) d v,
\end{aligned}
$$

which yield

$$
\frac{\partial F^{+}(p, p)}{\partial r}-\frac{\partial F^{+}(p, p)}{\partial p}
$$




$$
=-\frac{1}{2 \alpha}\left(q(p)+2 \int_{p}^{+\infty} q(u-p) H^{+}(u, p) d u+2 \int_{0}^{p} q(p-v) H^{+}(p, v) d v\right) .
$$

Therefore, from the first equality of $\left(2.52_{+}\right)$we find

$$
\begin{gathered}
\frac{\partial B^{+}(x, 0)}{\partial x} \\
=-\frac{1}{4}\left(q(p)+2 \int_{p}^{+\infty} q(u-p) H^{+}(u, p) d u+2 \int_{0}^{p} q(p-v) H^{+}(p, v) d v\right) .
\end{gathered}
$$

But then, by equation (2.5), the right-hand side of the last equality is equal to $\frac{\partial A^{+}(x, 0)}{\partial x}$. Therefore, we also have

$$
\frac{\partial B^{+}(x, 0)}{\partial x}=\frac{\partial A^{+}(x, 0)}{\partial x}
$$

In the similar way, taking into the account the integral equation for the kernel $A^{-}(x, t)$, from the second equalities of $(2.45),(2.46)$ and the first equality of $\left(2.52_{-}\right)$we obtain

$$
\frac{\partial B^{-}(x, 0)}{\partial x}=\frac{\partial A^{-}(x, 0)}{\partial x} .
$$

Now from formulas $\left(2.53_{ \pm}\right)$and $\left(2.54_{ \pm}\right)$we can conclude that the kernel functions $K^{ \pm}(x, t)$ and the derivatives $\frac{\partial K^{ \pm}(x, t)}{\partial x}$ are continuous at $x=0$.

Finally, from (2.51) and (2.52), we find that the kernels $B^{+}(x, t)$ and $B^{-}(x, t)$ satisfy the following partial differential equations:

$$
\begin{gathered}
\frac{\partial^{2} B^{+}(x, t)}{\partial x^{2}}-\alpha^{2} \frac{\partial^{2} B^{+}(x, t)}{\partial t^{2}}=q(x) B^{+}(x, t), \\
\frac{\partial^{2} B^{-}(x, t)}{\partial x^{2}}-\frac{\partial^{2} B^{-}(x, t)}{\partial t^{2}}=q(x) B^{-}(x, t) .
\end{gathered}
$$

From (2.13) and (2.24), it is easy to check that

$$
\begin{gathered}
\frac{\partial}{\partial r} F^{+}(r, 0)=-\frac{\alpha^{+}}{2 \alpha^{2}} q\left(\frac{r}{\alpha}\right), \\
\frac{\partial}{\partial p}\left[F^{+}\left(0^{+}, p\right)-F^{+}\left(0^{-}, p\right)\right]=-\frac{\alpha^{-}}{2 \alpha^{2}} q\left(-\frac{p}{\alpha}\right)
\end{gathered}
$$

and

$$
\begin{gathered}
\frac{\partial}{\partial r} F^{-}(r, 0)=\frac{\alpha \alpha^{+}}{2} q(r), \\
\frac{\partial}{\partial p}\left[F^{-}\left(0^{+}, p\right)-F^{-}\left(0^{-}, p\right)\right]=-\frac{\alpha \alpha^{-}}{2} q(p),
\end{gathered}
$$


which imply

$$
\begin{gathered}
\frac{d}{d x} B^{+}(x, \alpha x)=-\frac{\alpha^{+}}{2 \alpha} q(x) \\
\frac{d}{d x}\left\{B^{+}(x,-\alpha x+0)-B^{+}(x,-\alpha x-0)\right\}=\frac{\alpha^{-}}{2 \alpha} q(x)
\end{gathered}
$$

and

$$
\begin{gathered}
\frac{d}{d x} B^{-}(x, x)=\frac{\alpha \alpha^{+}}{2} q(x), \\
\frac{d}{d x}\left\{B^{-}(x,-x+0)-B^{-}(x,-x-0)\right\}=-\frac{\alpha \alpha^{-}}{2} q(x) .
\end{gathered}
$$

Hence we can formulate the following theorem:

Theorem 2. The kernel functions $K^{ \pm}(x, t)$ of integral representation (2.33) have both partial derivatives of the first order. If $q(x)$ is differentiable, then the kernel functions $K^{ \pm}(x, t)$ satisfy the partial differential equations

$$
\frac{\partial^{2} K^{ \pm}(x, t)}{\partial x^{2}}-\rho(x) \frac{\partial^{2} K^{ \pm}(x, t)}{\partial t^{2}}=q(x) K^{ \pm}(x, t), t \neq \mu(x)
$$

with conditions (2.34)-(2.37).

Note that equalities (2.34)-(2.37) can be written alternatively as

$$
\begin{gathered}
\frac{d}{d x} K^{+}(x, \mu(x))=-\frac{1}{4 \sqrt{\rho(x)}}\left(1+\frac{1}{\sqrt{\rho(x)}}\right) q(x), \\
\frac{d}{d x} K^{-}(x, \mu(x))=\frac{1}{4 \sqrt{\rho(x)}}\left(1+\frac{\alpha}{\sqrt{\rho(x)}}\right) q(x), \\
\frac{d}{d x}\left\{K^{+}(x,-\mu(x)+0)-K^{+}(x,-\mu(x)-0)\right\}=\frac{1}{4 \sqrt{\rho(x)}}\left(1-\frac{1}{\sqrt{\rho(x)}}\right) q(x), \\
\frac{d}{d x}\left\{K^{-}(x,-\mu(x)+0)-K^{-}(x,-\mu(x)-0)\right\}=\frac{1}{4 \sqrt{\rho(x)}}\left(1-\frac{\alpha}{\sqrt{\rho(x)}}\right) q(x) .
\end{gathered}
$$

Acknowledgment: The authors thank the referee for significant suggestions and remarks. 


\section{References}

[1] B.Ya Levin, Fourier and Laplace-Type Transformations by means of Solution of Second Order Differential Equation. — Dokl. Akad. Nauk SSSR 106 (1956), No. 2, 187-190. (Russian)

[2] V.A. Marchenko, Reconstruction of the Potential Energy From the Phases of the Scattered Waves. — Dokl. Akad. Nauk SSSR (Soviet Math. Dokl.) 104 (1955), 695698.

[3] L.D. Faddeev, The Inverse Problem in the Quantum Theory of Scattering. - Usp. Mat. Nauk 14 (1959), 57-119 [J. Math. Phys. 4 (1963), 72-104].

[4] B.M. Levitan, The Inverse Scattering Problem of Quantum Theory. - Math. Notes 17 (1975), 611-624.

[5] K. Chadan and P.C. Sabatier, Inverse Problems in Quantum Scattering Theory, Springer-Verlag, 1977.

[6] P. Deift and D. Trubowitz, Inverse Scattering on the Line. - Comm. Pure Appl. Math. 32 (1979), 121-251.

[7] V.A. Marchenko, Sturm-Liouville Operators and Their Applications. Basel, Birkhauser, 1986.

[8] B.M. Levitan, Inverse Sturm-Liouville Problems. Utrecht, VNU Science Press BV, 1987.

[9] I.M. Guseinov and R.T. Pashaev, On an Inverse Problem for a Second-Order Differential Equation. - Usp. Mat. Nauk 57 (2002), 147-148.

[10] Kh.R. Mamedov, On an Inverse Scattering Problem for a Discontinuous SturmLiouville Equation with a Spectral Parameter in the Boundary Condition. - Boundary Value Problems. Article ID 171967, 17 p., doi:10.1155/2010/171967 (2010). 